PROCEEDINGS OF THE

AMERICAN MATHEMATICAL SOCIETY

Volume 132, Number 1, Pages 223-230

S 0002-9939(03)07013-8

Article electronically published on June 12, 2003

\title{
SIMPLE CURVES IN $\mathbb{R}^{n}$ AND AHLFORS' SCHWARZIAN DERIVATIVE
}

\author{
MARTIN CHUAQUI AND JULIAN GEVIRTZ
}

(Communicated by Juha M. Heinonen)

\begin{abstract}
We derive sharp injectivity criteria for mappings $f:(-1,1) \rightarrow \mathbb{R}^{n}$ in terms of Ahlfors' definition of the Schwarzian derivative for such mappings.
\end{abstract}

\section{INTRODUCTION}

Because the Schwarzian derivative $S f=\left(f^{\prime \prime} / f^{\prime}\right)^{\prime}-\frac{1}{2}\left(f^{\prime \prime} / f^{\prime}\right)^{2}$ measures the extent to which an analytic function deviates from being a Möbius transformation, it carries information about both the local and global behavior of conformal mappings. Although in regard to the former $S f$ says something about how $f$ alters cross-ratios and curvature, the importance it has acquired in geometric function theory and related areas over the last 50 years or so stems primarily from Nehari's fundamental papers [Ne 1], Ne 2] on univalence criteria of the form

$$
|S f(z)| \leq 2 P(|z|)
$$

for analytic functions $f$ in the unit disk. In his most general version of this criterion [Ne 2], $P$ can be any even function for which (i) $\left(1-x^{2}\right)^{2} P(x)$ is nonincreasing on $[0,1)$, and (ii) the even solution of $U^{\prime \prime}+P U=0$ has no zeros. It is a straightforward consequence of condition (i) that (1.1) will imply univalence for any $P$ for which

$$
\varphi:(-1,1) \rightarrow \mathbb{C} \text { and }|S \varphi(x)| \leq 2 P(|x|) \Rightarrow \varphi \text { is injective, }
$$

so that the matter reduces in essence to showing that (1.2) holds under assumption (ii).

In this paper we shall give a very short proof that a stronger form of (1.2) actually holds under a weaker assumption on $P$, and more importantly, that such injectivity criteria hold for $f:(-1,1) \rightarrow \mathbb{R}^{n}$. In this wider context of curves in space we use a corresponding version of the Schwarzian due to Ahlfors [Ah], for which we offer a geometrically appealing definition, rather different in tenor from his, and which makes manifest that in this extended context, $S f$ continues to be a complex number invariant under Möbius transformations. Our analysis of the injectivity of $f$ and of the related issues of continuous extendibility to $[-1,1]$ and extremal behavior is based largely on an observation implicit in $[\mathrm{Ne} 2$ to the effect that it is only the real part of $S f$ that is of significance in such questions.

Received by the editors January 3, 2002 and, in revised form, September 5, 2002.

2000 Mathematics Subject Classification. Primary 53A04, 53A55; Secondary 30C55.

Key words and phrases. Schwarzian derivative, Nehari criteria, injectivity, extremal function. Both authors were partially supported by Fondecyt Grants \# 1000627 and \# 7000627. 


\section{HigheR-Dimensional CURVES}

In Ah] Ahlfors generalized the Schwarzian to cover $f:(a, b) \rightarrow \mathbb{R}^{n}$ by separately defining analogues of the 2-dimensional $\Re\{S f\}$ and $\Im\{S f\}$ as

$$
S_{1} f=\frac{\left\langle f^{\prime}, f^{\prime \prime \prime}\right\rangle}{\left|f^{\prime}\right|^{2}}-3 \frac{\left\langle f^{\prime}, f^{\prime \prime}\right\rangle^{2}}{\left|f^{\prime}\right|^{4}}+\frac{3}{2} \frac{\left|f^{\prime \prime}\right|^{2}}{\left|f^{\prime}\right|^{2}}
$$

and

$$
S_{2} f=\frac{f^{\prime} \wedge f^{\prime \prime \prime}}{\left|f^{\prime}\right|^{2}}-3 \frac{\left\langle f^{\prime}, f^{\prime \prime}\right\rangle}{\left|f^{\prime}\right|^{4}} f^{\prime} \wedge f^{\prime \prime},
$$

respectively. Here, for $\vec{a}, \vec{b} \in \mathbb{R}^{n}, \vec{a} \wedge \vec{b}$ is the antisymmetric bivector with components $(\vec{a} \wedge \vec{b})_{i j}=a_{i} b_{j}-a_{j} b_{i}$ and norm $\left(\sum_{i<j}\left(a_{i} b_{j}-a_{j} b_{i}\right)^{2}\right)^{1 / 2}$. Ahlfors indicated that he was led to these seemingly esoteric definitions by a direct identification of $\Re\{z \bar{\zeta}\}$ with the inner product $\langle z, \zeta\rangle$ of the 2-dimensional vectors $z, \zeta$ and the far from obvious identification of $\Im\{z \bar{\zeta}\}$ with the corresponding $\zeta \wedge z$ based on the fact that $(\Im\{z \bar{\zeta}\})^{2}=|\zeta \wedge z|^{2}$. In this section we give an equivalent but geometrically convincing derivation of what amounts to Alhfors' Schwarzian, very much in the spirit of his definition of the complex cross-ratio of four points in $\mathbb{R}^{n}$.

Let $C$ be a curve in $\mathbb{R}^{n}, n \geq 3$, parametrized by the $C^{3}$ function $f$ on $(a, b)$ with nonvanishing $f^{\prime}$. It is well-known that for each $t_{0} \in(a, b)$ on $C$, there is a $C^{\infty}$ function $g:(a, b) \rightarrow \mathbb{R}^{n}$ and a 2-sphere $K\left(t_{0}\right)$ (the osculating 2-sphere, which can degenerate into a plane; see, e.g., $[\mathrm{L})$ such that

$$
g((a, b)) \subset K\left(t_{0}\right)
$$

and

$$
f(t)=g(t)+o\left(\left|t-t_{0}\right|^{3}\right), t \rightarrow t_{0} .
$$

By regarding $K\left(t_{0}\right)$ as $\mathbb{C}$ via a stereographic projection, one can identify $g$ with a $\phi:(a, b) \rightarrow \mathbb{C}$, for which the expression $S \phi=\left(\phi^{\prime \prime} / \phi^{\prime}\right)^{\prime}-(1 / 2)\left(\phi^{\prime \prime} / \phi^{\prime}\right)^{2}$ of Section 2 is meaningful. In the case of a nondegenerate osculating sphere, one can take the vector from the point of contact to the center as $(0,0, R), R>0$, and give to the tangent plane, our $\mathbb{C}$, its usual (to be referred to as "canonical" below) orientation as $\mathbb{C}=\mathbb{R}^{2} \subset \mathbb{R}^{3}$. At points at which the osculating sphere degenerates to a plane, however, there is no canonical orientation for this plane, nor is there any canonical copy of $\mathbb{R}^{3}$ containing this plane. To circumvent this inherent ambiguity, we shall define $\mathcal{S} f\left(t_{0}\right)$ to be $S \phi\left(t_{0}\right)$ or $\overline{S \phi\left(t_{0}\right)}$, whichever one has a nonnegative imaginary part. Indeed, this is consistent with the cross-ratio $(\vec{a}, \vec{b}, \vec{c}, \vec{d})$ of $\vec{a}, \vec{b}, \vec{c}, \vec{d} \in \mathbb{R}^{n}$ as defined by Ahlfors in [Ah]: any given four points are always contained in a (possibly degenerate) 2-sphere $K$. One regards $K$ as $\mathbb{C}$, calculates the usual cross-ratio $k$, and gives to $(\vec{a}, \vec{b}, \vec{c}, \vec{d})$ the value $k$ or $\bar{k}$, whichever has a nonnegative imaginary part.

We show that $\mathcal{S} f\left(t_{0}\right)=S_{1} f\left(t_{0}\right)+i\left|S_{2} f\left(t_{0}\right)\right|$, thereby justifying the contention that the single complex number $\mathcal{S} f\left(t_{0}\right)$ embodies all of the information carried by Ahlfors' 2-part Schwarzian. We first consider the case of a nondegenerate osculating sphere. First of all, it is clear that both $S_{1} f\left(t_{0}\right)$ and $\left|S_{2} f\left(t_{0}\right)\right|$ remain unchanged when $f$ is replaced by $\rho U f+\vec{c}$, where $\rho \in \mathbb{R} \backslash\{0\}, U$ is a proper orthogonal transformation of $\mathbb{R}^{n}$, and $\vec{c} \in \mathbb{R}^{n}$ is a constant. Thus we may limit ourselves to the case in 
which $K\left(t_{0}\right)$ is the 2 -sphere contained in $\mathbb{R}^{3}=\left\{\left(x_{1}, x_{2}, x_{3}, 0, \ldots, 0\right): x_{1}, x_{2}, x_{3} \in\right.$ $\mathbb{R}\}$ with center at $(0,0,1)$. We denote by

$$
P(x+i y)=\left(\frac{2 x}{1+x^{2}+y^{2}}, \frac{2 y}{1+x^{2}+y^{2}}, 1-\frac{2}{1+x^{2}+y^{2}}\right)
$$

the usual stereographic projection of $\mathbb{C}$ onto the sphere in $\mathbb{R}^{3}$. In $\mathbb{R}^{3}$ the components of $\vec{a} \wedge \vec{b}$ are effectively those of $\vec{a} \times \vec{b}$. Let $h(t)=x(t)+i y(t)$, with $h(0)=0$. A straightforward, somewhat tedious calculation shows that

$$
S_{1}(P \circ h)(0)=\Re\{\mathcal{S h}(0)\}
$$

and

$$
S_{2}(P \circ h)(0)=(0,0, \Im\{\mathcal{S h}(0)\}) .
$$

In fact, these relations can be easily verified with any symbolic manipulation program, such as Maple or Mathematica, or even on a TI-92 calculator, since one can limit consideration to the case that $x$ and $y$ are cubic polynomials in $t$. From this the desired relation, $\mathcal{S} f\left(t_{0}\right)=S_{1} f\left(t_{0}\right)+i\left|S_{2} f\left(t_{0}\right)\right|$, follows immediately. In the case that the osculating sphere degenerates to a plane, by appropriate choices of $\rho, U$ and $\vec{c}$, we can arrange for this plane to be $\mathbb{R}^{2}=\left\{\left(x_{1}, x_{2}, 0, \ldots, 0\right): x_{1}, x_{2} \in \mathbb{R}\right\}$. Relations (2.4) and (2.5) again follow either by a limit argument or by direct calculation. We stress that in both cases the exact choice of $g$ is irrelevant since, in light of (2.3), only derivatives of order up to 3 enter into the calculations.

Theorem A. Let $f:(a, b) \rightarrow \mathbb{R}^{n}$ be a $C^{3}$ curve with nowhere vanishing $f^{\prime}$.

(a) For any Möbius transformation $T$ of $\mathbb{R}^{n}, \mathcal{S}(T \circ f)=\mathcal{S} f$.

$$
\begin{gathered}
\left(f\left(t_{0}+t \alpha\right), f\left(t_{0}+t \beta\right), f\left(t_{0}+t \gamma\right), f\left(t_{0}+t \delta\right)\right) \\
=(\alpha, \beta, \gamma, \delta)\left[1+\frac{1}{6}(\alpha-\beta)(\gamma-\delta) \mathcal{S}^{*} f\left(t_{0}\right) t^{2}\right]+o\left(t^{2}\right), \text { as } t \rightarrow 0,
\end{gathered}
$$

where $\mathcal{S}^{*} f$ is $\mathcal{S} f$ or its conjugate according to whether $(\alpha, \beta, \gamma, \delta)(\alpha-\beta)(\gamma-\delta)$ is nonnegative or not.

Comment. Conclusion (i) implies that $\mathcal{S} f$ has meaning for $C^{3}$ mappings $f$ : $(a, b) \rightarrow \mathbb{R}^{n} \cup\{\infty\}=\mathbb{S}^{n} \subset \mathbb{R}^{n+1}$. Conclusion (ii) extends a similar relation involving Ahlfors' $S_{1} f=\Re\{\mathcal{S} f\}$.

Proof. (a) For $t_{0} \in(a, b)$ let $K\left(t_{0}\right)$ be the corresponding osculating sphere and let $g=g(t)$ be as in (2.3). The Möbius transformation $T$ will carry $K\left(t_{0}\right)$ onto the osculating 2-sphere of $T \circ f$ at $T \circ f\left(t_{0}\right)$, at which point this curve has contact of order 3 with $T \circ g$. According to our definition, $\mathcal{S} f\left(t_{0}\right)$ and $\mathcal{S}(T \circ f)\left(t_{0}\right)$ are interpreted as complex numbers after stereographically projecting the respective curves $g$ and $T \circ g$ onto the complex plane. Because $T$ is Möbius, it is clear that the two stereographic projections are related by a planar Möbius mapping, which will preserve the Schwarzian as defined.

(b) To show this, observe that the relevant terms in the expansion considered will remain unchanged if we replace $f$ by $g$. After a suitable stereographic projection of the curve given by $g$, we can assume that we are working in $\mathbb{C}$. This formula is valid with $f$ replaced by $g$ and $\mathcal{S}^{*} f$ by $S g$. The desired conclusion now follows by replacing the imaginary parts on both sides by their absolute values. 
Going back to relations (2.1) and (2.2), $S_{1} f$ and $S_{2} f$ can be written in terms of the geometry of the trace of $f$. We write

$$
f^{\prime}=v \hat{t} \quad \text { and } \quad f^{\prime \prime}=v^{\prime} \hat{t}+v^{2} k \hat{n}
$$

where $v>0$ and $\hat{t}, \hat{n}$ are the unit tangent and normal vectors. A third differentiation gives

$$
f^{\prime \prime \prime}=v^{\prime \prime} \hat{t}+v v^{\prime} k \hat{n}+2 v v^{\prime} k \hat{n}+v^{2} k^{\prime} \hat{n}+v^{2} k \hat{n}^{\prime} .
$$

Since $\hat{n}$ is a unit vector, $\left\langle\hat{n}^{\prime}, \hat{n}\right\rangle=0$, and upon differentiating $\langle\hat{t}, \hat{n}\rangle=0$ we see that the component of $\hat{n}^{\prime}$ in the direction of $\hat{t}$ must equal $-v k$. Thus the equation

$$
\hat{n}^{\prime}=-v k \hat{t}+v \tau \hat{b}
$$

defines both the binormal vector $\hat{b}$ and the torsion $\tau$. From this we obtain

$$
f^{\prime \prime \prime}=\left(v^{\prime \prime}-v^{3} k^{2}\right) \hat{t}+\left(3 v v^{\prime} k+v^{2} k^{\prime}\right) \hat{n}+v^{3} k \tau \hat{b}
$$

so that

$$
S_{1} f=\frac{v^{\prime \prime}-v^{3} k^{2}}{v}-3 \frac{\left(v^{\prime}\right)^{2}}{v^{2}}+\frac{3}{2} \frac{\left(v^{\prime}\right)^{2}+v^{4} k^{2}}{v^{2}}=\left(\frac{v^{\prime}}{v}\right)^{\prime}-\frac{1}{2}\left(\frac{v^{\prime}}{v}\right)^{2}+\frac{1}{2} v^{2} k^{2} .
$$

Thus, if $s(x)$ denotes arc length, then

$$
S_{1} f=S s(x)+\frac{1}{2} v^{2} k^{2} .
$$

Although it will not be used in the sequel, we derive a corresponding formula for $S_{2} f$. It follows from the expressions given above for $f^{\prime}, f^{\prime \prime}$ and $f^{\prime \prime \prime}$ that

$$
f^{\prime} \wedge f^{\prime \prime}=v^{3} k(\hat{t} \wedge \hat{n}) \quad \text { and } \quad f^{\prime} \wedge f^{\prime \prime \prime}=v^{2}\left(3 v^{\prime} k+v k^{\prime}\right)(\hat{t} \wedge \hat{n})+v^{4} k \tau(\hat{t} \wedge \hat{b}) .
$$

A computation gives that

$$
\langle\vec{a} \wedge \vec{b}, \vec{a} \wedge \vec{c}\rangle=|\vec{a}|^{2}\langle\vec{b}, \vec{c}\rangle-\langle\vec{a}, \vec{b}\rangle\langle\vec{a}, \vec{c}\rangle,
$$

which implies that in the $(n(n-1) / 2)$-dimensional space, $\hat{t} \wedge \hat{n}$ and $\hat{t} \wedge \hat{b}$ are orthonormal. With this we now write

$$
S_{2} f=\left(3 v^{\prime} k+v k^{\prime}\right)(\hat{t} \wedge \hat{n})+v^{2} k \tau(\hat{t} \wedge \hat{b})-3 v^{\prime} k(\hat{t} \wedge \hat{n})=v k^{\prime}(\hat{t} \wedge \hat{n})+v^{2} k \tau(\hat{t} \wedge \hat{b}) .
$$

\section{INJECTIVITY CRITERIA AND EXTENDIBILITY}

In several places in the proofs to follow, we make use of the classical Sturm comparison theorem, which we state here for reference.

Theorem. Let $u, v$ be positive functions on $(a, b)$ which satisfy $u^{\prime \prime}+p u=0, v^{\prime \prime}+$ $q v=0$, where $p \leq q$, and $u\left(x_{0}\right)=v\left(x_{0}\right), u^{\prime}\left(x_{0}\right)=v^{\prime}\left(x_{0}\right)$ for some $x_{0} \in(a, b)$. Then $u \geq v$ on $(a, b)$.

For convenience, we use Ahlfors' original notation $S_{1} f$ for $\Re\{\mathcal{S} f\}$.

Theorem B. Let $P=P(x)$ be a continuous function defined on $(-1,1)$ with the property that no nontrivial solution $u$ of $u^{\prime \prime}+P u=0$ has more than one zero. Let $f:(-1,1) \rightarrow \mathbb{R}^{n} \cup\{\infty\}$ be a curve of class $C^{3}$ with nowhere vanishing $f^{\prime}$. If $S_{1} f(x) \leq 2 P(x)$ on $(-1,1)$, then $f$ is one-to-one. 
Proof. If not, then $f\left(x_{1}\right)=f\left(x_{2}\right)$ for $x_{1}<x_{2}$ in $(-1,1)$, where $f$ is one-to-one on $\left[x_{1}, x_{2}\right)$. Let $g=T \circ f$ be a Möbius transformation of $f$ that takes $f\left(x_{1}\right)$ to the point at infinity, and let $v=\left|g^{\prime}\right|^{-1 / 2}$. Then $v$ is regular in the open interval $\left(x_{1}, x_{2}\right)$, and a simple calculation shows that $v^{\prime \prime}+q v=0$, where

$$
2 q=\frac{\left\langle g^{\prime}, g^{\prime \prime \prime}\right\rangle}{\left|g^{\prime}\right|^{2}}+\frac{\left|g^{\prime \prime}\right|^{2}}{\left|g^{\prime}\right|^{2}}-\frac{5}{2} \frac{\left\langle g^{\prime}, g^{\prime \prime}\right\rangle^{2}}{\left|g^{\prime}\right|^{4}}=S_{1} g-\frac{1}{2}\left(\frac{\left|g^{\prime \prime}\right|^{2}}{\left|g^{\prime}\right|^{2}}-\frac{\left\langle g^{\prime}, g^{\prime \prime}\right\rangle^{2}}{\left|g^{\prime}\right|^{4}}\right) \leq S_{1} f,
$$

hence $q \leq P$. A suitable solution $U_{1}$ of $U^{\prime \prime}+P U=0$ coincides with $v$ to first order at some point $x_{0} \in\left(x_{1}, x_{2}\right)$, so that by the Sturm comparison theorem, $v(x) \geq U_{1}(x)$ on the interval containing $x_{0}$ where $U_{1}(x) \geq 0$. Since by hypothesis $U_{1}$ has at most one zero in the interval $(-1,1)$, we conclude that $v$ has a positive lower bound in a neighborhood of either $x_{1}$ or $x_{2}$. But then $\left|g^{\prime}\right|$ will be bounded above in that neighborhood, making it impossible for $g$ to become infinite there.

In light of (2.6) we have

Corollary C. Let $P$ be as in the previous theorem and let $f:(-1,1) \rightarrow \mathbb{R}^{n}$ be an arclength parametrized curve with geodesic curvature $k$. If $k^{2}(s) \leq 4 P(s)$ on $(-1,1)$, then $f$ is one-to-one.

Interesting examples such as

$$
P(x)=\frac{\pi^{2}}{4}, \frac{1}{\left(1-x^{2}\right)^{2}}, \frac{2}{1-x^{2}},
$$

can be obtained from conditions for univalence of analytic functions in the disk $\mathbb{D}=\{|z|<1\}$. For these choices the criteria $|S f(z)| \leq 2 P(|z|)$ in $\mathbb{D}$ admit extremal functions that are unique up to Möbius transformations and which map the interval $[-1,1]$ onto a closed curve. We shall show that no new extremal functions appear for these criteria in the context of curves in $\mathbb{R}^{n}$. Although not necessary, to make the discussion of this point as simple as possible, we will assume that $P(x)$ is an even function. This implies that the solution $U_{0}$ of $U^{\prime \prime}+P U=0$ with initial conditions $U_{0}(0)=1, U_{0}^{\prime}(0)=0$ is also even, and hence can have no zeros on $(-1,1)$ since otherwise it would have at least two. We define

$$
F(x)=\int_{0}^{x} U_{0}^{-2}(t) d t
$$

so that $F$ is odd and satisfies $S F=2 P, F(0)=0, F^{\prime}(0)=1, F^{\prime \prime}(0)=0$. When we regard $F$ as a mapping of $(-1,1)$ into $\mathbb{R} \subset \mathbb{R}^{n} \cup\{\infty\}$, the mappings $T \circ F$ with $T$ Möbius are precisely those that manifest extremal behavior. More precisely, we have

Theorem D. Let $f:(-1,1) \rightarrow \mathbb{R}^{n} \cup\{\infty\}$ satisfy $f(0)=0,\left|f^{\prime}(0)\right|=1, f^{\prime \prime}(0)=0$ and suppose that $S_{1} f(x) \leq 2 P(x)$. Let $P$ be as in Theorem $\mathrm{B}$, and in addition be even. Then

(a) $\left|f^{\prime}(x)\right| \leq F^{\prime}(|x|)$ on $(-1,1)$ and $f$ admits a (spherically) continuous extension to $[-1,1]$.

(b) If $F(1)<\infty$, then $f$ is one-to-one on $[-1,1]$ and $f([-1,1])$ has finite length.

(c) If $F(1)=\infty$, then either $f$ is one-to-one on $[-1,1]$ or, up to rotation, $f=F$.

Proof. It is not difficult to see that the normalization assumed in the statement can always be achieved by composing $f$ with a suitable Möbius transformation. Indeed, if we map the osculating sphere of $f$ at $f(0)$ onto a 2-dimensional subspace 
$\mathbb{R}^{2}$ (regarded as $\mathbb{C}$ ) with a Möbius transformation $T$, then, after replacing $f$ by $T \circ f$, we can regard $f(0), f^{\prime}(0)$, and $f^{\prime \prime}(0)$ as complex numbers. After suitable translation, rotation and dilation, we can then obtain $f(0)=0, f^{\prime}(0)=1$, and $f^{\prime \prime}(0)=2 \alpha$. Composition of the extension to $\mathbb{R}^{n}$ of the Möbius map $z /(1+\alpha z)$ of the plane with this $f$ results in one with the desired properties. Again let $v=\left|f^{\prime}\right|^{-1 / 2}$. As pointed out in the proof of Theorem B, $v^{\prime \prime}+q v=0$ for some $q \leq P$, and because of the normalization of $f, v(0)=1, v^{\prime}(0)=0$. Thus the Sturm comparison theorem implies that $v(x) \geq U_{0}(x)$, so that $\left|f^{\prime}(x)\right| \leq F^{\prime}(|x|)$.

If $F(1)<\infty$, then both integrals

$$
\int_{0}^{1}\left|f^{\prime}(x)\right| d x \quad, \quad \int_{-1}^{0}\left|f^{\prime}(x)\right| d x
$$

are finite, which implies that $f$ admits a continuous extension to $[-1,1]$ and that $f([-1,1])$ has finite length.

Suppose that $F(1)=\infty$, and let $G(y)=F^{-1}(y),-\infty<y<\infty$. We consider the function

$$
w(y)=\left(\frac{v}{U_{0}}\right)(G(y)) .
$$

Since $G^{\prime}(y)=U_{0}^{2}(G(y))$, it follows easily that

$$
w^{\prime \prime}=(P-q) U_{0}^{4} w
$$

where $P, q, U_{0}$ are evaluated at $G(y)$. Also, $w(0)=1, w^{\prime}(0)=0$. Because $2 q \leq$ $S_{1} f \leq 2 P, w$ is convex. We claim that on each of the half-intervals $(-1,0]$ and $[0,1$ ), either $f=F$ (up to rotation), or else $f$ can be extended to the endpoint so that the image of that half has finite length. The analysis being the same for each half, we consider $[0,1)$. If $q<P$ at a single point, then $w(y) \geq a y+b, a>0$ for all large $y$. Hence for $x$ close to 1

$$
\left|f^{\prime}(x)\right|=v^{-2}(x) \leq \frac{U_{0}^{-2}(x)}{(a F(x)+b)^{2}}=\frac{F^{\prime}(x)}{(a F(x)+b)^{2}}=-\frac{1}{a} \frac{d}{d x}\left(\frac{1}{a F(x)+b}\right),
$$

which implies that $\int_{0}^{1}\left|f^{\prime}\right| d x<\infty$, so that $f([0,1))$ once again has finite length, and $f$ admits a continuous extension to $[0,1]$. On the other hand, it follows from (3.1) that $q \equiv P$ on $[0,1)$ only if $S_{1} f=2 P$ and $f^{\prime}, f^{\prime \prime}$ are linearly dependent. But then $f$ maps that half onto a line, and because of the normalization at the origin and the fact that $S_{1} f=P$ it follows that, up to a rotation, $f=F$, and again we have a spherically continuous extension. This completes the proof of (a).

It remains only to show that this continuous extension to $[-1,1]$ is injective except in the case of (c) when $f$ coincides with the extremal $F$ on the entire interval. If $f$ is not one-to-one, then either $f(1)=f(-1)$ or there exists $x_{0} \in(-1,1)$ such that $f\left(x_{0}\right)$ equals, say $f(1)$ (the case $f\left(x_{0}\right)=f(-1)$ being the same except for notational details). Thus, in either case there exists $x_{0} \in[-1,1)$ such that $f\left(x_{0}\right)=f(1)$ and $f$ is one-to-one on $\left[x_{0}, 1\right)$. Let $T$ once again be a Möbius transformation such that $g=T \circ f$ satisfies $g(1)=\infty$. Then $v=\left|g^{\prime}\right|^{-1 / 2}$ is regular on $\left(x_{0}, 1\right)$ and satisfies $v^{\prime \prime}+q v=0$, where $2 q \leq S_{1} f \leq 2 P$ as in (3.1). It is easily verified that the general solution of $U^{\prime \prime}+P U=0$ is $\alpha U_{0}+\beta U_{0} F=(\alpha+\beta F) U_{0}$. Let $c=\left(1+x_{0}\right) / 2$. 
If we choose $a, b$ such that $v(c), v^{\prime}(c)$ coincide with the corresponding values for $(a+b F) U_{0}$, then by Sturm comparison, $v \geq(a+b F) U_{0}$ on any subinterval of $\left(x_{0}, 1\right)$ containing $c$ on which $(a+b F) U_{0}$ is positive. Since $F$ is increasing and $a+b F(c)=v(c) / U_{0}(c)>0, a+b F$ will have to be positive on at least one of $\left(x_{0}, c\right)$ or $(c, 1)$. Then on this interval

$$
\left|g^{\prime}\right| \leq \frac{1}{(a+b F)^{2} U_{0}^{2}}=\frac{F^{\prime}}{(a+b F)^{2}},
$$

so that we will have $\int_{c}^{1}\left|g^{\prime}\right| d x<\infty$ or $\int_{x_{0}}^{c}\left|g^{\prime}\right| d x<\infty$ (contradicting of the fact that $\left.g\left(x_{0}\right)=g(1)=\infty\right)$, unless $b=0, x_{0}=-1$ and $F(1)=F(-1)=\infty$. Since in this case $g(-1)=g(1)=\infty$, we can replace $g$ by a multiple of it so that $v(0)=1$ (and $\left.v^{\prime}(0)=0\right)$. We again consider the convex function $w$ defined in (3.2), and recall that the analysis leading to (3.3) shows that $g$ cannot be infinite at both 1 and -1 unless $S_{1} g=2 P$ and $g((-1,1))$ is a straight line. Because $g=T \circ f$ and $f(0)=0,\left|f^{\prime}(0)\right|=F^{\prime}(0)$, and $f^{\prime \prime}(0)=F^{\prime \prime}(0)$, it is clear that $f$ is a rotation of $F$.

\section{Final COMMENTS}

1. The situation considered in part (b) of Theorem D is essentially the case of a nonsharp univalence criterion. More precisely, it can be shown in this case that when $\left(1-x^{2}\right)^{2} P(x)$ is nonincreasing there exists $\lambda>1$ such that $S_{1} f \leq 2 \lambda P$ still implies injectivity [Ch]. We also point out that Theorem D is a curve analogue of a theorem of Gehring and Pommerenke [Ge-Po.

2. The Schwarzian for curves as presented in Section 2 makes sense for $C^{3}$ curves in a Hilbert space of arbitrary dimension, since the osculating sphere remains meaningful in that context. Indeed, the normalizing procedures, as well as the inversion operation taking a point to infinity, used in the proofs are well-defined and continue to leave the Schwarzian unaltered. For this reason, Theorems A, B, $\mathrm{C}$, and D carry over verbatim.

3. Since, as indicated in the Introduction, injectivity for curves based on bounds on $S f$ translates into injectivity for conformal mappings, Theorem B should have a counterpart for $F: D \rightarrow \mathbb{R}^{n}$, for appropriate domains $D \subset \mathbb{R}^{n}$, and indeed it does, if one is content with bounds on $S F$ calculated in all directions. It is easy to see, for example, how this would work for convex $D$, in which case an optimal bound would be $2 \pi^{2} /(\operatorname{diam} D)^{2}$. It would be nice, however, to find a more elegant statement to this effect, based perhaps on a bound for a single expression involving partial derivatives of order up to 3 of $F$.

\section{REFERENCES}

[Ah] L. V. Ahlfors, Cross-ratios and Schwarzian derivatives in $\mathbb{R}^{n}$, Complex Analysis, 1-15, Birkhäuser, Basel, 1988. MR 90a:30055

[Ch] M. Chuaqui, On a theorem of Nehari and quasidiscs, Ann. Acad. Sci. Fenn., Ser. A.I. Math. 18 (1993), 117-124. MR 94f:30026

[Ge-Po] F. W. Gehring and Ch. Pommerenke, On the Nehari univalence criterion and quasicircles, Comment. Math. Helv. 59 (1984), 226-242. MR 85f:30023

[L] D. Laugwitz, Differential and Riemannian Geometry, Academic Press, New York, 1965. MR 30:2406 
[Ne 1] Z. Nehari, The Schwarzian derivative and schlicht functions, Bull. Amer. Math. Soc. 55 (1949), 545-551. MR 10:696e

[Ne 2] Z. Nehari, Univalence criteria depending on the Schwarzian derivative, Illinois J. Math. 23 (1979), 345-351. MR 80i:30033

Facultad de Matemáticas, P. Universidad Católica de Chile, Casilla 306, Santiago 22, CHILE

E-mail address: mchuaqui@mat.puc.cl

Facultad de Matemáticas, P. Universidad Católica de Chile, Casilla 306, Santiago 22, CHILE

E-mail address: jgevirtz@mat.puc.cl 